\title{
Análisis de los diferentes métodos de enseñanza utilizados en el fútbol base Analysis of different teaching methods in young soccer players
}

\author{
Norberto Pascual Verdú, Eduardo NicolásAlzamora Damiano, JoséA. Martínez Carbonell y JoséA. Pérez Turpin \\ Universidad de Alicante (España)
}

Resumen: La utilización de un método de enseñanza u otro es un aspecto importante en la formación de jóvenes jugadores de fútbol. El objetivo del presente estudio ha sido identificar y analizar los métodos de enseñanza que utilizan los entrenadores en el fútbol de iniciación. Para ello, se analizaron a 36 entrenadores de fútbol 8 de categorías benjamín y alevín del campeonato de la Comunidad Valenciana (liga 2013-2014), mediante una encuesta diseñada y validada. Los resultados obtenidos, muestran que el método de enseñanza más utilizado es el método global, siendo la combinación de varios métodos de enseñanza la forma más idónea para enseñar el fútbol.

Palabras clave: Fútbol, Fútbol base, métodos de enseñanza, entrenadores de fútbol.

Abstract: The use of a teaching method or another is an important aspect in training of young soccer players. The aim of study was to identify and analyze the teaching methods coaches use in young soccer players. Analysis of 36 soccer coaches of 8 soccer categories 9-10 years and 11-12 years of Valencia Community Championship (League 2013-2014), using a validated questionnaire. The results indicate that the teaching method most often used by coaches is the global method and that the combination of several teaching methods is the most appropriate way to teach football.

Key Words: Soccer, young soccer players, teaching methods, soccer coaches.

\section{Introducción}

Cada entrenador utiliza los métodos de enseñanza que cree más convenientes para formar a los jugadores que tiene a su cargo. Sin embargo, la elección de estos métodos se ve muchas veces condicionada por el club en el que trabaja o por las características de sus jugadores, así como por otros aspectos como las instalaciones o los recursos humanos y materiales de los que dispone. Pero trabajar en el fútbol base también exige a los entrenadores estar en un proceso de formación continuo, donde en muchas ocasiones el entrenador tiene que conocer el desempeño de la labor de otros profesionales para poder valorar y mejorar su propia actuación, con el fin de aumentar la calidad de sus sesiones de entrenamientos y en consecuencia, la de sus jugadores.

Si bien los partidos de competición son un gran escenario para el aprendizaje, es en las sesiones de entrenamiento donde el entrenador propone los ejercicios y selecciona los métodos de enseñanza que él cree más adecuados para formar a sus jugadores.

Según Pacheco (2004), «en el proceso de la enseñanza/aprendizaje del fútbol, se debe intentar utilizar métodos de enseñanza eficaces, es decir, aquellos que permitan asimilar más y mejor los aprendizajes». Una revisión bibliográfica sobre los métodos de enseñanza que son utilizados actualmente en el fútbol de formación, nos acerca a definir principalmente los siguientes métodos:

- Método Analítico. Consiste en presentar la tarea descompuesta por partes aisladas del juego real, habitualmente a modo de repeticiones. El juego se descompone de forma que solo se tienen en cuenta algunos elementos y aspectos (normalmente técnica con balón). Ejemplo: En parejas, pases y controles.

- Método Global o Integral. Consiste en presentar una situación de juego real de forma completa donde intervienen varios elementos (balón, reglamento, compañeros, adversarios...) y varios aspectos (entrenadores, tácticos, preparación física....). Ejemplo: Juego reducido (posesión) 3x3.

- Método Mixto. Consiste en combinar el método analítico y el método global. La ejecución de este método se comienza con un ejercicio global, después se realiza un ejercicio analítico, y se termina volviendo a un ejercicio global. La secuencia sería: Tarea Global-TareaAnalíticaTarea Global. Ejemplo: Juego reducido (posesión) 7x7-Regate 1x1Juego reducido (posesión) 7x7 prestando atención en el regate.

- Método Sistémico. Consiste en presentar actividades como un todo, en las que se lleva a cabo un proceso cognitivo donde el jugador es el verdadero protagonista del aprendizaje. El jugador interviene activamente en la toma de decisiones. Ejemplo: Salida de balón desde portería.

Fecha recepción: 13-02-15- Fecha envío revisores: 18-02-15- Fecha de aceptación: 26-03-15 Norberto Pascual Verdú norberto.pascual@ua.es
- Otros métodos. Además de los métodos antes mencionados, es posible que algún entrenador use personalmente otros métodos de enseñanza o algunos métodos creados por el mismo, que nada tienen que ver con los ya comentados anteriormente, por ello, a fin de aunar criterios, a estos métodos los englobaremos bajo el término de «otros métodos».

Una vez definidos los métodos de enseñanza, somos conscientes de que posiblemente algunos entrenador/entrenadores, debido a su nivel de formación, no conozcan los métodos como tal, aunque seguramente los utilicen de forma indirecta o inconsciente en sus entrenamientos.

Según Pascual (2012), «la utilización de un método u otro sólo puede justificarse si se analiza específicamente el contenido/objetivo del entrenamiento a desarrollar en cada momento, el nivel y la fase en la que se encuentran los jóvenes deportistas (iniciación, perfeccionamiento, rendimiento)». Por ello, cabe mencionar que en este estudio solo se abarcan las principales categorías de formación del denominado fútbol 8 (Benjamín y Alevín), en las que se engloban jugadores entre los 8 y los 12 años que se encuentran en la fase de iniciación, donde ya están preparados para iniciar el aprendizaje de las destrezas técnicas del juego individual y de los conocimientos tácticos del juego colectivo.

El objetivo de este estudio es conocer qué métodos de enseñanza se están utilizando actualmente en las diferentes categorías de fútbol de formación (Benjamín y Alevín) y el resultado de los mismos.

\section{Metodología}

\section{Participantes}

El estudio se realizó a 36 entrenadores de fútbol, de género masculino, que desarrollaban su labor en las categorías Benjamín (n=23) y

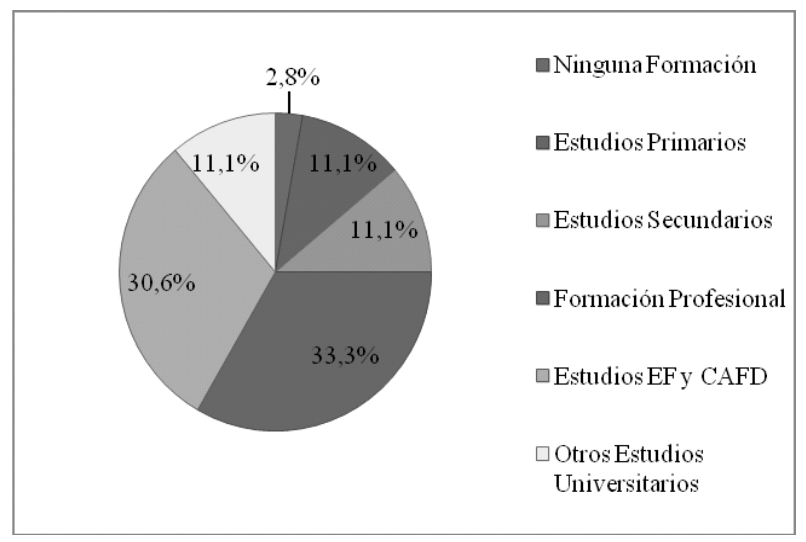

Figura 1. Máxima formación académica de los entrenadores. 
Alevín (n=13), en 12 clubs federados de fútbol de la Comunidad Valenciana durante la temporada 2013 - 2014.

En referencia a su máxima formación académica-véase figura 1-, indicaron que el 2.8\% no tenía ninguna formación académica, el 11.1\% tenía estudios primarios completos (certificado escolar), el 11.1\% tenía estudios secundarios, bachillerato o B.U.P. y C.O.U., el 33.3\% tenía un título de formación profesional, el 30.6\% tenía estudios universitarios relacionados con la educación física y las ciencias de la actividad física y el deporte, y el 11.1\% tenía otros estudios universitarios.

En cuanto a su máxima formación específica en fútbol-véase figura 2-, el 33.3\% manifestaron no poseer este tipo de formación, mientras que el $66.7 \%$ sí declararon tenerla. Con respecto a su nivel de formación, el 30.6\% declararon poseer el Curso de Iniciación Aspirante a Técnico Deportivo de Fútbol (Monitor), el 13.9\% indicaron tener el título de Entrenador de Fútbol de Primer Nivel (Nivel 1), el 13.9 señalaron tener el título de Entrenador de Fútbol de Segundo Nivel (Nivel 2) y el 8.3\% manifestaron poseer el título de Entrenador de Fútbol de Tercer Nivel (Nivel 3).

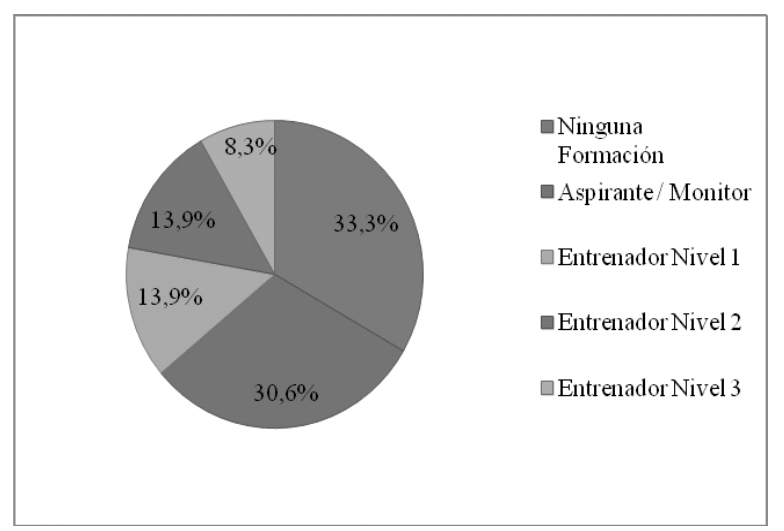

Figura 2. Máxima fomación de fútbol de los entrenadores.

\section{Instrumentos}

Para la recogida de datos se diseñó una encuesta, que tras ser validada, fue enviada a los diferentes entrenadores de fútbol. Esta encuesta fue confeccionada teniendo en cuenta tanto la revisión bibliográfica, como las aportaciones por parte de un grupo de expertos.

La encuesta tenia la siguiente estructura: un primer apartado con información general acerca de la procedencia de la encuesta (entrenador encuestado, equipo, categoría y e-mail); un apartado de cinco preguntas de respuesta única que hacía referencia a datos personales sobre los entrenadores (género, edad, años de experiencia, formación académica y formación específica de fútbol); un apartado, a modo de información, donde se encontraba una descripción de los diferentes métodos de enseñanza en el fútbol; un apartado de seis preguntas en relación a los métodos de enseñanza en el fútbol, combinando preguntas de respuesta única y otras de respuesta múltiple; y un último apartado con una pregunta que hacía referencia a los porcentajes (\%) de métodos de enseñanza en el fútbol que utilizaban los entrenadores durante una sesión de entrenamiento.

Para la validación del contenido de la encuesta, tras la elaboración de un primer borrador, se envió a un grupo de expertos constituido por seis doctores en Ciencias de la Actividad Física y el Deporte, que impartían asignaturas del Grado en Ciencias de la Actividad Física y el Deporte en la Universidad deAlicante y que además eran entrenadores de diferentes deportes, incluyendo el fútbol. Posteriormente se realizaron las modificaciones pertinentes para validar la encuesta.

\section{Análisis estadístico de los datos}

Los análisis de datos se han realizado mediante la utilización de la hoja de cálculo del programa Microsoft Office Excel versión 2007. Los resultados fueron obtenidos tras realizar un análisis cuantitativo empleando los estadísticos frecuencia $(\mathrm{N})$ y porcentaje $(\%)$. Al tratarse de un análisis descriptivo comparativo se emplearon gráficos de barras y gráficos de sectores para facilitar la comprensión de los resultados.

\section{Resultados}

En relación al conocimiento de los métodos de enseñanza en el fútbol - véase figura 3-, los resultados obtenidos tras el análisis de las encuestas indican que los métodos analítico y global o integral son los más conocidos por parte de los encuestados (83.3\%). Algo menos conocido es el método que combina los dos anteriores, hablamos del método mixto, el cual es conocido por el $80.6 \%$ de los entrenadores. El método menos conocido es el sistémico, que tan solo es conocido por el $69.4 \%$ de los encuestados. También cabe destacar que el $11.1 \%$ de los entrenadores señalaron conocer otros métodos de enseñanza diferentes a los expuestos en la encuesta. Por su parte, una pequeña minoría del 8.3\% indicó no conocer ningún método de enseñanza de fútbol.

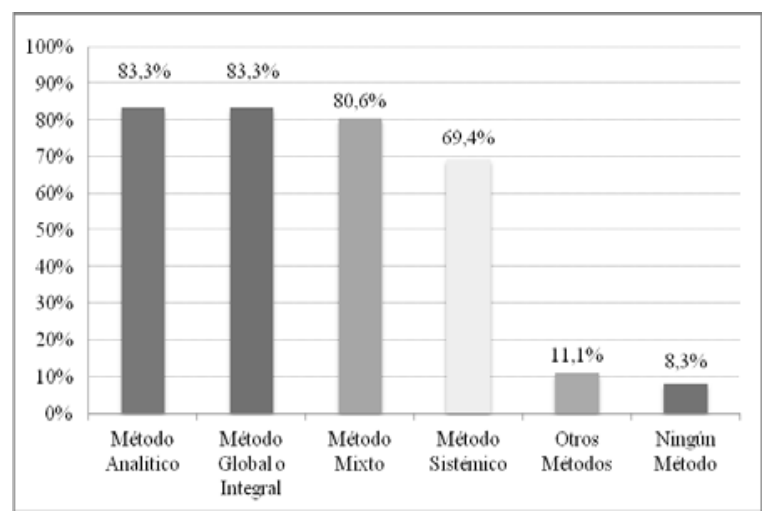

Figura 3. Conocimiento de cada uno de los métodos de enseñanza de fútbol por parte de los entrenadores.

En cuanto a los métodos de enseñanza de fútbol que son utilizados por parte de los entrenadores encuestados -véase figura 4-, los resultados muestran que el método de enseñanza analítico es utilizado por el $63.9 \%$ de los entrenadores, mientras que el método global o integral es utilizado en mayor porcentaje por el 83.3\%. También son utilizados el método mixto, por el $72.2 \%$ de los encuestados y el método sistémico, que es utilizado en menor medida por $58.3 \%$ de los entrenadores. Por otro lado, una minoría del 8.3\% de los entrenadores, afirman utilizar otros métodos de enseñanza diferentes a los anteriores.

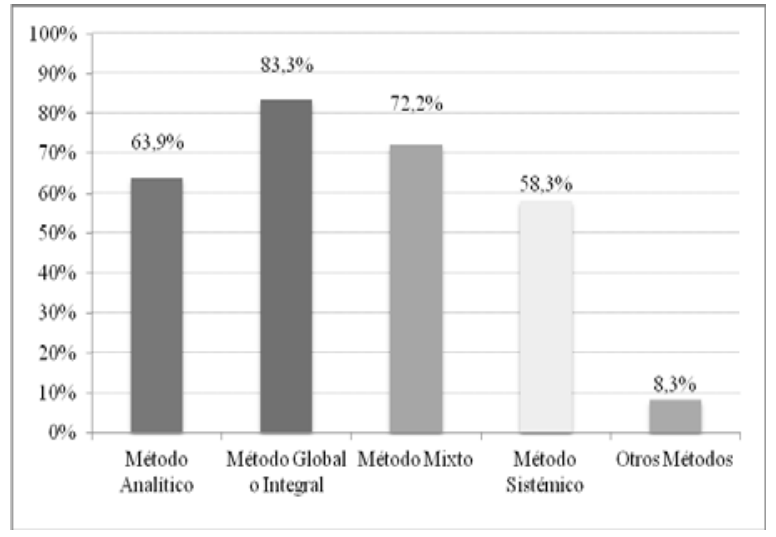

Figura 4. Métodos de enseñan za que son utilizados por parte de los entrenadores encuestados.

En referencia a cual es el método de enseñanza más utilizado por cada entrenador-véase figura 5-, el 61.1\% señalaron que el método que más utilizan es el global o integral. Seguidamente, el método más utilizado por los entrenadores es el mixto 27.8\%. Además, el $11.1 \%$ de los encuestados indicaron que el método de enseñanza que más utilizaban era el método sistémico. Ninguno de los encuestados señaló que el método que más utilizaba era el método analítico.

Asimismo, la encuesta incluía una pregunta acerca de cuáles eran los 


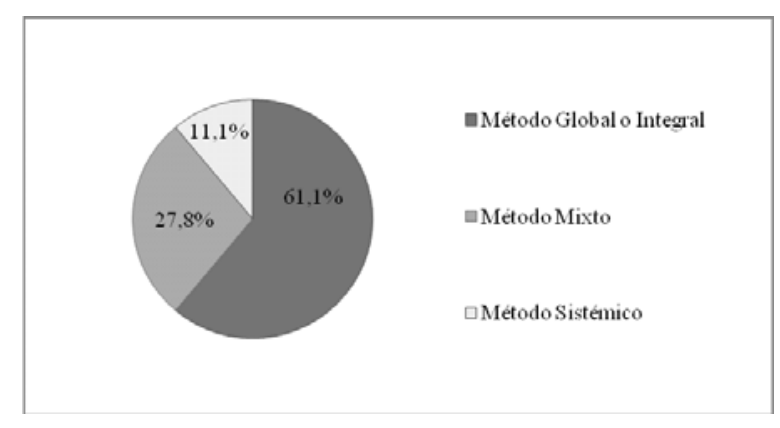

Figura 5. M étodos de enseñanza de fútb ol más utilizados por parte de los entrenadores.

parámetros que influían en la elección de un método de enseñanza u otro por parte del entrenador. Con relación a estos parámetros, el 44.4\% de los entrenadores indicó que la utilización de su método de enseñanza estaba basada en las características de los jugadores. Del mismo modo, el $41.7 \%$ señaló que uno de los parámetros que influían en la elección del método de enseñanza eran los contenidos y objetivos de la sesión. Además, la filosofía del club fue uno de los parámetros señalado por el $27.8 \%$ de los entrenadores. De la misma manera, el $19.4 \%$ de los encuestados afirman basar su método de enseñanza en función de las instalaciones y los recursos materiales y humanos disponibles. Asimismo, el 25\% de los entrenadores afirman utilizar su método de enseñanza en función de otros parámetros.

Por otra parte, se preguntó a cada entrenador cuál era el método de enseñanza que creía que era más conveniente utilizar para la categoría en la que entrenaba. En relación a las respuestas de los 23 entrenadores de la categoría Benjamín se puede indicar que el 26.1\% de estos entrenadores eligieron el método global o integral como el más adecuado para la categoría Benjamín. Otro 26.1\% de los encuestados se decantó por el método mixto como el más conveniente para esta categoría. Asu vez, un 8.7\% de entrenadores escogieron como método más eficaz el método sistémico. Sin embargo, el 39.1\% de los entrenadores señalan que la combinación de varios métodos es el modo más idóneo para la enseñanza en la categoría Benjamín.

Por otro lado, en cuanto a los resultados de las respuestas de los 13 entrenadores de la categoría Alevín, se observa que el 7.7\% escogen el método analítico como más idóneo para la enseñanza en esta categoría. Mientras que el 23.1\% apuestan por el método global o integral, el $30.8 \%$ lo hacen por el método mixto. Por su parte, un $7.7 \%$ eligen el método sistémico como el más adecuado en esta etapa. Asimismo, el $30.8 \%$ de los entrenadores encuestados de la categoría Alevín creen que lo más conveniente para la enseñanza a estas edades es la combinación de varios métodos.

Una última cuestión, de forma global, hacía referencia a los porcentajes (\%) que se utilizan para cada método de enseñanza durante una sesión de entrenamiento (figura 6). Los resultados obtenidos fueron los siguientes: método analítico 16.1\%, método global o integral 38.2\%, método mixto $23.1 \%$, método sistémico $18.3 \%$ y otros métodos $4.3 \%$.

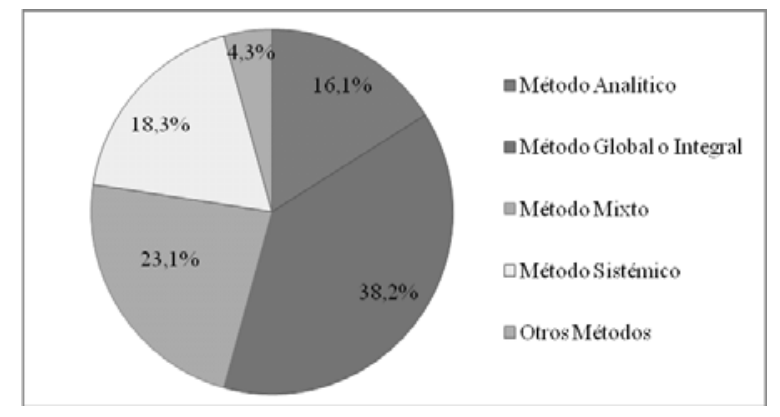

Figura 6. Porcentajes (\%) de métodos de en señanza utilizados por cada entrenador durante una sesión.

\section{Discusión}

Al parecer, tras el análisis de los resultados obtenidos, el método global o integral es el más utilizado por los entrenadores de fútbol que ejercen su labor en categorías de formación. Quizás esto se debe a que, como mencionan Sans y Frattarola (2007), «el método global incide simultáneamente en aspectos técnicos, tácticos, y físicos, lo que permite, durante un juego, desarrollarlos todos en forma conjunta, aunque sea de una manera genérica». Por su parte, Lasierra (1993), citado por Gómez y Salavert(2013) indica que «la práctica global es solidaria con el aprendizaje, ya que el niño en cualquier situación interviene de forma global y, por tanto, necesita de esa práctica más que de ejercicios «nocivamente analíticos». Además, teniendo en cuenta las características del entrenamiento en estas categorías, donde la mayor parte de los equipos entrenan entre $2-3$ horas semanales, es posible que la mayor utilización del método global se deba al insuficiente tiempo de entrenamiento disponible, donde se necesita trabajar una gran multitud de aspectos. Aquí, volvemos a mencionar, esta vez en una publicación anterior, a Sans y Frattarola (2000), donde en su libro Entrenamiento del Fútbol Base nos indican que «el método global permite obtener un resultado más eficaz utilizando el escaso tiempo de que disponemos para formar a nuestros jóvenes futbolistas».

Por el contrario, el método analítico, a pesar de ser muy conocido, es menos utilizado por parte de los entrenadores del fútbol base, lo que contradice a lo publicado por Rui Pacheco (2004), cuando refiriéndose al método analítico en su libro La enseñanza y entrenamiento en el fútbol 7 menciona que «el método de enseñanza basado en la técnica es aquel que aún hoy en día impera en nuestros clubes», claro está que esta afirmación es del año 2004. Sin embargo, coincidimos con este autor, cuando señala que «el tipo de enseñanza, donde se realizan ejercicios de una forma aislada de las situaciones reales de juego, hace muy difícil mantener durante largos períodos de tiempo la motivación y el rendimiento de los jugadores, ya que las situaciones propuestas son poco estimulantes por no poseer los aspectos esenciales del juego». Por su parte, Ardá y Casal (2003) postulan que «la automatización de cualquier acción a través de la reproducción y repetición en condiciones analíticas no garantiza su correcta realización en el juego». Estos pueden ser algunos de los motivos por el cual este método sea menos utilizado por los entrenadores de categorías de formación.

En relación a los métodos mixto y sistémico, a pesar de ser algo menos conocidos, los resultados muestran que también son comúnmente utilizados por parte de los entrenadores encargados de la formación en fútbol base.

Con respecto a estos dos últimos métodos, es poca la bibliografía que encontramos para poder realizar una discusión acerca de ellos, con el fin de contrastar nuestros resultados con las perspectivas de otros autores, ya que la mayoría de autores se centran en comparar el método analítico con el método global. Así Sans y Frattarola (2000) señalan que «tradicionalmente, ha existido un enfrentamiento teórico entre la elección del método global y el analítico como fundamento en la enseñanza del fútbol».

A pesar de que el método global es el más utilizado por parte de los entrenadores encargados de la formación en las categorías inferiores del fútbol base, tras nuestro estudio podemos indicar que los entrenadores creen más conveniente que el entrenamiento en categorías Benjamín y Alevín se lleve a cabo combinando varios métodos de enseñanza. De este modo, coincidimos con Yagüe et al. (2012) en señalar que «la planificación del aprendizaje deportivo debería combinar la estrategia analítica y global, pues para introducir al niño en el manejo de habilidades menos comunes hay que emplear el programa técnico, mientras que para mejorar la motricidad vinculada al lado dominante debe emplearse el programa táctico». Asimismo, Sans y Frattarola (2000), también refiriéndose a estos dos métodos, terminan expresando que «la elección de un método no implica la marginación del otro, que más bien para la formación completa del jugador se requiere la utilización de ambos, aunque con un porcentaje menor el método analítico». Al mismo tiempo, estos autores agregan que «el método global, no es suficiente para la formación completa del joven jugador. Así, el programa también contempla la utilización del método analítico, aunque en porcentaje inferior». De similar manera, Koch (1996) plantea que «el entrenador solo 
será equitativo cuando coordine el método global con el método analítico. Ambos métodos tienen que utilizarse paralelamente».

En cuanto a los parámetros que influyen en la elección de un método de enseñanza u otro, al parecer tanto las características de los jugadores, como los objetivos y contenidos de la sesión de entrenamiento son los parámetros principales que sirven para seleccionar el método de enseñanza que principalmente se va a utilizar.

En este sentido, estamos de acuerdo con Pascual (2012) en afirmar que «el debate acerca del método de enseñanza/entrenamiento a desarrollar en el fútbol es algo más complejo que la simple elección de unos en detrimento de otro/s. La utilización de un método u otro sólo puede justificarse si se analiza específicamente el contenido/objetivo del entrenamiento a desarrollar en cada momento, el nivel y la fase en la que se encuentran los jóvenes deportistas (iniciación, perfeccionamiento, rendimiento). En cada caso lo que habrá que hacer será una utilización preferencial, que no exclusiva, de un método sobre los otros».

Según Seirul-lo (1999), «en deportes como el nuestro, también cobran gran importancia mecanismos cognitivos vinculados a la percepción, interpretación, decisión y planificación motriz». Todos estos aspectos produjeron la necesidad de definir un nuevo método de enseñanza que fuera más allá de los aspectos técnicos, tácticos y físicos. Con estas reflexiones, se establece el método sistémico. De estemodo, Pascual (2012) señala que «estos presupuestos han llevado a la implantación de un nuevo método de enseñanza del juego, basado en la búsqueda dirigida y en los juegos condicionados como juego de fútbol en los que se ponen algunos condicionantes para alcanzar determinados objetivos en el juego (método sistémico), intentando formar jugadores inteligentes, capaces de responder de una forma adecuada a las diferentes situaciones del juego».

Según nuestro estudio, el método sistémico es el que menos se conoce y utiliza por parte de los entrenadores en categorías de formación. Por ello, esto contradice a Garganta(1998), mencionado por Sánchez (2012), cuando sostiene que «en los juegos deportivos colectivos, estamos asistiendo a una transición de los modelos analítico y estructuralista para un modelo sistémico».

\section{Conclusiones}

A continuación, se presentan las conclusiones obtenidas en el presente estudio:

- El método de enseñanza global o integral es más utilizado que el analítico por los entrenadores en categorías de formación (Benjamín y Alevín).

- El método sistémico, debido a su desconocimiento, es poco utilizado por los entrenadores de fútbol base.

- La combinación de varios métodos de enseñanza es la forma más idónea para la enseñanza del fútbol.

\section{Referencias}

Abad Robles, M.T. (2010). La formación del entrenador en el proceso de enseñanza-aprendizaje de jóvenes futbolistas. Tesis doctoral. Universidad de Huelva, Huelva.

Abad Robles, M.T., Giménez Fuentes-Guerra, F.J., Robles Rodríguez, J. \& Rodríguez López, J.M. (2011). Perfil, experiencia y métodos de enseñanza de los entrenadores de jóvenes futbolistas en la provincia de Huelva. Revista Retos. Nuevas tendencias en Educación Física, Deporte y Recreación, 20, 21-25.

Arda, T. \& Casal, C. (2003). Metodología de enseñanza del fútbol. Barcelona: Paidotribo.

Ashley, C., Méndez Giménez, A. \& Valero Valenzuela, A. (2010). What are we being told about how to teach games? A three-dimensional analysis of comparative research into different instructional studies in Physical Education and School Sports. International Journal of Sport Science, 18, 37-56.

Brüggemann, D. (2004). Entrenamiento para niños y jóvenes. Barcelona: Paidotribo.
Delgado Noguera, M.A. \& Sicilia Camacho, Á. (2002). Educación física y estilos de enseñanza. Barcelona: Inde.

Díaz de Rada Igúzquiza, V. (2009). Análisis de datos de encuesta. Barcelona: Editorial UOC.

Gómez Galiana, S. \& Salavert Hervás, D. (2013). Metodología de la enseñanza y del entrenamiento del fútbol. Generalitat Valenciana: Enseñanzas Deportivas.

Koch, W. (1996). Fútbol base. Programas de entrenamiento (14 - 15 años). Barcelona: Paidotribo.

Lledó Figueres, E. \& Huertas Olmedo, F. (2012). Perfil del técnico de fútbol en escuelas de clubes de primera división en la Comunitat Valenciana. Revista Apunts, Educación Física y Deportes, 108, 3545.

Pascarella, J. (2013). The French elite formation coaching license methodology (whole, part, whole) vs the USSF coaching methodology (simple to complex). World Class Coaching Magazine, October 2013 Issue.

Pascual Verdú, N. (2012). Análisis comparativo de los sistemas de juego en fútbol siete alevín. Tesis doctoral. Universidad de Alicante, Alicante.

Pazo Haro, C. I. (2011). La formación de los jugadores de fútbol de la alta competición desde la perspectiva de los coordinadores de cantera. Revista Apunts, Educación Física y Deporte, 104, 56-65.

Ramírez Molina, S. (2011). Metodología del fútbol base del CD Alcoyano. Alcoy: Área de metodología del CD Alcoyano.

Rivas Borbón, M. (2013). Guía didáctica para la enseñanza del fútbol en niños y adolescente de 8 a 16 años. Universidad Nacional de Costa Rica: Escuela de ciencias del movimiento humano y calidad de vida.

Rui Pacheco (2004). La enseñanza y entrenamiento del fútbol 7. Un juego de iniciación al fútbol 11. Barcelona: Paidotribo.

Ruiz de Alarcón Quintero, A., Reina Gómez, Á., Fernández García, J.C. \& Beas Martínez, M.A. (2006). Análisis de la iniciación al fútbol. Lecturas de educación física. Efdeportes, 92. Consultado el 21 de abril del 2014 de http://www.efdeportes.com/efd92/inic.htm

Sánchez López de Toro, R. (2012). La triada «paradigmas, modelos, metodologías». Hacia una amputación (y reconstrucción) de términos ambiguamente utilizados en el estudio del fútbol. Lecturas de educación física. Efdeportes, 164. Consultado el 14 de mayo del 2014 de http://www.efdeportes.com/efd164/terminos-ambiguamente-utilizados-en-el-futbol.htm

Sans Torrelles, A. \& Frattarola Alcaraz, C. (2000). Entrenamiento en el fútbol base.

Barcelona: Paidotribo.

Sans Torrelles, A. \& Frattarola Alcaraz, C. (2007). Manual para la organización y el entrenamiento en las escuelas de fútbol. Barcelona: Paidotribo.

Seirul-lo, F. (1999). Criterios modernos de entrenamiento del fútbol. Revista Training Fútbol, 45, 8-18.

Valero Valenzuela, A. (2005). Análisis de los cambios producidos en la metodología de la iniciación deportiva. Revista Apunts, Educación Física y Deporte, 79, 59-67.

Vegas Haro, G (2006). Metodología de enseñanza basada en la implicación cognitiva del jugador de fútbol base. Tesis doctoral. Universidad de Granada, Granada.

Vegas Haro, G, Romero Rodríguez. R.C. \& Pino Ortega, J. (2012). Metodología de enseñanza en el fútbol basada en la implicación cognitiva del jugador. Sevilla: Wanceulen.

Viciana, J. \& Mayorga-Vega, D. (2014). Differences between tactical/ technical models of coaching and experience on the instructions given by youth soccer coaches during competition. Journal of Physical Education and Sport, 14(1), 3-11.

Yagüe Cabezón, J.M., Sánchez Sánchez, J. \& Molinero. O. (2012). Incidencia de dos metodologías de entrenamiento-aprendizaje sobre la técnica individual de futbolistas de 6 a 10 años de edad. Revista Retos. Nuevas tendencias en Educación Física, Deporte y Recreación, 22, 29-32.

Yagüe Cabezón, J.M., Sánchez Sánchez, J. \& Veroz Domínguez, R. (2004). Estudio sobre la metodología de entrenamiento y las fuentes de formación permanente que utilizan los entrenadores de la cantera de un club de fútbol profesional. Universidad de León, León. 